\title{
Brinquedo terapêutico: análise do comportamento e da dor de crianças no primeiro curativo pós-operatório
}

Therapeutic play: analysis of children's behavior and pain in the first postoperative dressing Juego terapéutico: análisis del comportamiento y dolor de los niños en el primer apósito postoperatorio

Ludmilla Laura Miranda ${ }^{1}$ (D) https://orcid.org/0000-0001-8777-1745

Mauren Teresa Grubisich Mendes Tacla ${ }^{1}$ (D) https://orcid.org/0000-0001-8928-3366

Flávia Lopes Gabani ${ }^{1}$ (D) https://orcid.org/0000-0002-9442-4896

Rosângela Aparecida Pimenta Ferrari' ${ }^{1}$ (D) https://orcid.org/0000-0003-0157-7461

\section{Resumo}

Objetivo: Analisar o comportamento e dor de crianças submetidas ao primeiro curativo após procedimento cirúrgico.

Métodos: Trata-se de estudo observacional, realizado em unidade pediátrica de hospital universitário público, de dezembro de 2016 a agosto 2017. Foram observadas 112 crianças durante o primeiro curativo pós-operatório utilizando-se a Escala de Comportamento e a Escala de Faces de Dor de Claro/FPS-R. Os dados foram analisados no programa SPSS ${ }^{\circ}$ em frequências e proporções.

Resultados: Do total, 58,0\% tinham sete anos ou mais de idade, quase $83,0 \%$ referiram dor durante 0 curativo, sendo $61,5 \%$ de intensidade moderada a insuportável. Crianças submetidas à apendicectomia demonstraram menor prevalência dos comportamentos analisados durante a sessão de brinquedo terapêutico. Também foi um dos procedimentos com maior incidência de dor insuportável durante 0 curativo.

Conclusão: A dor foi frequentemente referida durante a primeira troca de curativo, independentemente da idade, sexo e tipo de procedimento cirúrgico, com repercussões no comportamento infantil.

\section{Abstract}

Objective: To analyze the behavior and pain of children submitted to the first dressing after a surgical procedure. Methods: This is an observational study, carried out in a pediatric unit of a public university hospital, from December 2016 to August 2017. 112 children were observed during the first postoperative dressing using the Behavior Scale and the Faces Scale of Claro's pain / FPS-R. The data were analyzed using the SPSS ${ }^{\circledR}$ program in frequencies and proportions.

Results: $58.0 \%$ were seven years of age or older, almost $83.0 \%$ reported pain during the dressing, $61.5 \%$ of moderate to unbearable intensity. Children who underwent appendectomy showed a lower prevalence of the behaviors analyzed during the therapeutic play session. It was also one of the procedures with the highest incidence of unbearable pain during the dressing.

Conclusion: Pain was frequently referred to during the first dressing change, regardless of age, sex and type of surgical procedure, with repercussions on children's behavior.

\section{Resumen}

Objetivo: analizar el comportamiento y el dolor de los niños sometidos al primer vendaje después del procedimiento quirúrgico.

Métodos: Este es un estudio observacional, llevado a cabo en una unidad pediátrica de un hospital universitario público, de diciembre de 2016 a agosto de 2017. Se observaron 112 niños durante el primer vendaje postoperatorio utilizando la Escala de Comportamiento y la Escala de Rostros de El dolor de Claro / FPS-R. Los datos se analizaron utilizando el programa SPSS ${ }^{\circledR}$ en frecuencias y proporciones.

Resultados: $58.0 \%$ tenían siete años de edad o más, casi $83.0 \%$ reportó dolor durante el vendaje, $61.5 \%$ de intensidad moderada a insoportable. Los niños que se sometieron a apendicectomía mostraron una menor prevalencia de los comportamientos analizados durante la sesión de juego terapéutico. También fue uno de los procedimientos con mayor incidencia de dolor insoportable durante el vendaje.

Conclusión: se hizo referencia al dolor con frecuencia durante el primer cambio de apósito, independientemente de la edad, el sexo y el tipo de procedimiento quirúrgico, con repercusiones en el comportamiento de los niños.

\section{Descritores}

Dor pós-operatória; Criança;

Hospitalização; Enfermagem pediátrica

\section{Keywords}

Pain, postoperative; Child; Hospitalization; Pediatric nursing

\section{Descriptores}

Dolor postoperatorio; Niño; Hospitalización; Enfermería pediátrica

\section{Como citar:}

Miranda LL, Tacla MT, Gabani FL, Ferrari RA. [Therapeutic play: analysis of children's behavior and pain in the first postoperative dressing]. Rev Soc Bras Enferm Ped. 2020;20(1):13-21. Portuguese

${ }^{1}$ Departamento de Enfermagem, Centro de Ciências da Saúde, Universidade Estadual de Londrina, Londrina, PR, Brasil.

Conflitos de interesse: nada a declarar.

Submetido: 13 de Maio de 2019 | Aceito: 22 de Junho de 2020

Autora correspondente: Ludmilla Laura Miranda | Email: m.ludmilla@hotmail.com

DOI: http://dx.doi.org/10.31508/1676-3793202000003 


\section{Introdução}

A hospitalização na infância é considerada momento muito difícil por causar, na criança, sentimentos de ordens física e emocional, expondo-a à dor, impotência e incapacidades. ${ }^{(1,2)}$ Nesse período, muitas delas são submetidas a procedimentos cirúrgicos, o que implica em lesão de tecidos, com consequente dor pós-operatória intensa. ${ }^{(1-3)}$

Procedimentos potencialmente frequentes durante a hospitalização incluem desde administração de medicamentos até realização de cirurgias de grande porte, sendo necessários, muitas vezes, curativos, injeções, punções venosas, biópsias, drenagens de líquidos corporais, entre outras intervenções que geram dor, ansiedade e podem adquirir caráter ameaçador, agressivo e invasivo. Apesar desses eventos serem comumente observados em internações, a dor, em especial na criança, é prejudicial e causa diversos danos que podem ser evitados frente aos vários meios para identificá-la e minimizá-la. ${ }^{(4-7)}$

De acordo com o Conselho Nacional dos Direitos da Criança e do Adolescente, Resolução 41 que dispõe sobre os Direitos da Criança e Adolescente Hospitalizados, afirma no artigo $7^{\circ}$ que o paciente tem "direito a não sentir dor quando existem meios para evitá-la". ${ }^{(2)} \mathrm{A}$ dor pós-operatória é provável, mas pode não ocorrer se for vista como prioridade no planejamento terapêutico de pacientes pediátricos. Se tratada preventivamente e de forma precoce, menos traumas serão gerados na criança, com maior possibilidade de colaboração da mesma durante a realização de curativos, minimizando sofrimento, mudanças bruscas de comportamento (reações de agressividade e choro intenso), e repercussões sistêmicas (aumento das frequências cardíaca e respiratória, e da pressão arterial). ${ }^{(5,6)}$ Preparar a criança antes da execução de procedimentos também pode colaborar nesse processo. ${ }^{(7,8)}$

A dor é subjetiva e, quando se trata de avaliação e quantificação, é imprescindível compreendê-la adequadamente conforme recomendações já validadas em literatura. Existem métodos multidimensionais de avaliação da dor (escalas) utilizados e recomendados para obter informações individualizadas das crianças. São instrumentos padronizados que consideram as peculiaridades das faixas etárias pediátricas. ${ }^{(2,5,6)}$
Além da avaliação da dor, os profissionais também podem utilizar a avaliação do comportamento infantil durante intervenções, observando alterações nas expressões faciais, corporais e/ou verbais. Essa estratégia é capaz de identificar mudanças comportamentais e avaliar a qualidade efetiva do manejo realizado. ${ }^{(7)}$

Nem sempre as unidades hospitalares possuem uma dinâmica diferenciada capaz de receber e preparar previamente a criança para um procedimento cirúrgico e cuidados pós-operatórios. Não é raro situações cirúrgicas emergenciais, indisponibilidade de leitos em unidades de internação e complexo gerenciamento das salas cirúrgicas conforme prioridade do diagnóstico, fatos que dificultam contato e estabelecimento de confiança com a equipe de saúde que receberá a criança para cuidados pós-operatórios, gerando tensões, medos e ansiedades sobre o que está por vir. Uma estratégia para aliviar essas angústias é a utilização do brincar como forma de trazer o mundo lúdico ao ambiente hospitalar. Por meio da brincadeira a criança é capaz de expressar emoções, receber instruções e demonstrar inseguranças e incertezas, desde que seja realizada seguindo técnicas específicas, tendo como instrumento de educação o brinquedo terapêutico (BT). ${ }^{(1,4,8-10)}$

Assim, o presente estudo teve por objetivo descrever como a criança em pós-operatório se comporta durante a realização do primeiro curativo, incluindo a mensuração da dor durante o procedimento, utilizando como técnica a aplicação do BT.

\section{Métodos}

Trata-se de estudo quantitativo, realizado numa unidade pediátrica de hospital universitário público de alta complexidade. O hospital presta assistência a crianças de zero a 12 anos de idade para tratamentos cirúrgicos e clínicos, com disponibilidade total de 20 leitos na enfermaria de pediatria.

Os critérios de inclusão foram: crianças entre quatro e 12 anos, idade em que foi possível aplicar a escala de dor elegida para uso neste estudo, e que não receberam nenhum preparo prévio ao procedimento cirúrgico, sendo admitidas na unidade pediátrica após saída do centro cirúrgico; ter seus curativos realizados nas primeiras 24 horas de pós- 
-operatório, pela maior estimativa de intensidade de dor, nos turnos matutino ou vespertino, independente se em dias úteis ou dias de final de semana, por serem períodos em que havia enfermeiros residentes em Saúde da Criança capacitados para observar o procedimento conforme o método desta pesquisa; ter condições cognitivas para responder a intensidade da dor visualizando a Escala de Face de Dor de Claro; e ter a autorização dos pais ou responsáveis para participarem do estudo mediante assinatura do Termo de Consentimento Livre e Esclarecido. Além disso, o profissional responsável por realizar o primeiro curativo (enfermeiro ou auxiliar/técnico de enfermagem) precisava aceitar a observação dos enfermeiros residentes (dois) durante sua execução.

Entre dezembro de 2016 e agosto de 2017, período do presente estudo, a unidade pediátrica atendeu 464 crianças, das quais 194 foram após procedimentos cirúrgicos. Conforme os critérios de inclusão, restaram para compor a população desta pesquisa 112 crianças.

A coleta de dados ocorreu durante a realização do primeiro curativo utilizando-se um checklist que avalia o comportamento da criança (Escala de Comportamento) e a intensidade da dor (Escala de Faces de Dor de Claro/FPS-R). ${ }^{(6,7)}$ Para preencher o cheklist da Escala de Comportamento, o enfermeiro residente, que desenvolve suas atividades práticas na unidade pediátrica, programou com o profissional responsável pela criança o horário do curativo que, de acordo com a rotina da unidade, é realizado logo após o banho, nas primeiras 24 horas de pós-operatório. A observação da criança iniciou-se no momento em que o profissional entrava na enfermaria com o material de curativo para abordagem, preparo e realização do procedimento, que durava de 10 a 20 minutos. Ao término do curativo aplicava-se a escala de dor com objetivo de registrar a intensidade da dor sentida pela criança. Vale ressaltar que a unidade em estudo utiliza a Escala de Faces de Dor de Claro (Cebolinha) para registro da dor como $5^{\mathbf{o}}$ sinal vital desde 2007.

Esperava-se a criança se restabelecer e, em momento oportuno, era realizado convite para participar de uma sessão de BT. O objetivo da sessão era captar as reações e percepções da criança sobre os procedimentos a que foi submetida.
Para a sessão de BT foram utilizados os seguintes materiais: boneco, luvas, talas, gorro, máscara, campos cirúrgicos, tesoura, pinças de curativo, equipamentos que representassem um ambiente hospitalar, almotolia com soro fisiológico, gaze, esparadrapo ou fita adesiva similar, e outros itens específicos conforme o tipo de intervenção sofrida pela criança.

Os pesquisadores, inclusive, preparavam previamente o boneco tentando deixá-lo o mais próximo possível das características físicas da criança, com inserções de curativos e dispositivos hospitalares. A criança era instruída sobre o funcionamento da sessão, devendo a mesma devolver os brinquedos após seu término, o que geralmente demorava de 30 a 40 minutos.

Enquanto um enfermeiro residente aplicava a sessão de BT, o outro realizava a observação da criança conforme o checklist da Escala do Comportamento. Os comportamentos abordados no instrumento são (presente ou ausente): observa, manuseia, brinca, interage com o adulto, verbaliza, recusa, sorri, demonstra, expressa tristeza, medo ou insatisfação, compara, repete ações e movimentos rituais. Já a Escala de Faces, adaptada por Claro no Brasil, é composta por cinco expressões do personagem Cebolinha, da Turma da Mônica, que variam sem dor até a dor insuportável $(0=$ sem dor, $1=$ dor leve, 2 = dor moderada, 3 = dor forte $4=$ dor insuportável), autorreferida pelo próprio paciente. ${ }^{(6)}$ É aplicável às crianças a partir dos três anos de idade durante a fase de hospitalização. Essa escala é de fácil administração e não requer equipamento excepcional, exceto as "faces" fotocopiadas.

Para o estudo foram selecionadas as seguintes variáveis: faixa etária, sexo, diagnóstico de internação conforme a Classificação Internacional de Doenças, décima versão (CID-10), procedimento cirúrgico realizado, tempo de internação, comportamento observado durante a troca de curativo e escore de dor segundo a Escala de Faces de Dor de Claro.

Para análise estatística de comparação das variáveis categóricas utilizou-se o programa Statistical Package for the Social Sciences (SPSS ${ }^{\circledR}$ ), considerando o teste exato de Fisher pelo tamanho amostral reduzido, sendo significativo quando $p<0,05$. Cada variável do comportamento foi analisada individualmente e de forma dicotômica (presente ou au- 
sente), de acordo com a faixa etária (4 a 6 anos e $\geq$ 7 anos), sexo (masculino e feminino) e diagnóstico pré-operatório/procedimento (sim e não). A mesma consideração foi feita na análise do escore de dor com faixa etária, sexo e comportamento, com a diferença do escore de dor ser categorizado de intensidade zero a quatro.

A pesquisa foi autorizada pelas diretorias do hospital e aprovada pelo Comitê de Ética em Pesquisa Envolvendo Seres Humanos da Universidade Estadual de Londrina parecer $\mathrm{n}^{\mathrm{o} 039 / 2014}$, CAAE $\mathrm{n}^{\circ}$ 27836414900005231.

\section{Resultados}

Das 112 crianças incluídas neste estudo, 54,4\% eram meninos e $58,0 \%$ tinham sete anos ou mais de idade. Os principais diagnósticos cirúrgicos de internação abrangeram os sistemas gastrointestinal (50,8\%), respiratório $(16,9 \%)$ e geniturinário $(16,0 \%)$. Quanto aos procedimentos cirúrgicos destacaram-se apendicectomia $(30,3 \%)$, drenagens de pneumotórax e derrame pleural decorrentes de complicações de pneumonias $(16,9 \%)$, uretroplastia por anomalias geniturinárias $(16,0 \%)$, drenagem percutânea de abscesso $(8,0 \%)$, herniorrafia inguinal bilateral $(8,0 \%)$ e colostomia por obstrução intestinal não especificada $(8,0 \%)$.

Cerca de 5,0\% das crianças submetidas à laparotomia a causa foi apendicite com peritonite generalizada. Apendicite foi mais frequente em meninos (51,3\%), e derrame pleural mais comum em meninas $(57,9 \%)$. Quanto à faixa etária, o primeiro diagnóstico prevaleceu entre crianças com sete anos ou mais (76,9\%), e o segundo entre aquelas com menos de sete anos de idade $(63,2 \%)$.

O tempo de internação variou de dois a 32 dias, em que $47,3 \%$ das crianças ficaram de dois a cinco dias internadas, e 27,6\% mais de 11 dias. No comportamento das crianças durante a realização do primeiro curativo, $74,1 \%$ observaram e presenciaram o profissional preparar os materiais para o procedimento, 65,0\% manusearam previamente os materiais, $66,0 \%$ interagiram com o profissional ou acompanhante, e $23,2 \%$ brincaram e conversaram durante a técnica. Cerca de $44,0 \%$ não queriam realizar o procedimento, $68,7 \%$ expressaram sentimentos de tristeza, medo e insatisfação, e 41,0\% demonstraram movimentos rituais, ou seja, de defesa, e inquietação. Quase 83,0\% referiram dor durante o curativo, sendo $61,5 \%$ de intensidade moderada a insuportável.

Quando à sessão de BT, houve diferença estatisticamente significativa do comportamento presente referente ao primeiro curativo pós-operatório das crianças conforme a faixa etária, com maior participação das menores de sete anos de idade. Observar, manusear, interagir com adulto, verbalizar, recusar a realização do curativo, demonstrar o procedimento, expressar tristeza, medo e insatisfação, e realizar movimentos rituais durante a sessão de BT, no boneco, foram observados em mais de $90,0 \%$ das crianças menores de sete anos. Nas maiores de sete anos prevaleceu o ato de observar $(59,7 \%)$ e demonstrar o procedimento $(59,7 \%)$. Não houve diferença significativa em relação ao sexo. Em relação aos procedimentos cirúrgicos, verificou-se que crianças submetidas à apendicectomia demonstraram menor prevalência dos comportamentos analisados durante a sessão de BT, no boneco, variando de $19,4 \%$ a $38,9 \%$, seguidas das crianças que realizaram uretroplastia, que apresentou prevalência acima do 90,0\% somente no comportamento observar (Tabela 1).

Em relação ao escore de dor (Tabela 2), não houve diferença estatística significativa da faixa etária e sexo de acordo com a intensidade da dor referida logo após o primeiro curativo pós-operatório. A dor classificada como forte (escore 3) prevaleceu em crianças submetidas à herniorrafia $(55,6 \%)$, seguidas por uretroplastia $(44,4 \%)$ e drenagem percutânea $(44,4 \%)$. Já a classificada como insuportável (escore 4), esteve mais presente naquelas que realizaram apendicectomia com peritonite generalizada $(60,0 \%)$ e drenagem torácica (52,6\%). Quanto aos comportamentos observados na criança com seu boneco durante a sessão de BT segundo intensidade da dor autorreferida, destacaram-se observar, manusear, interagir com adulto, verbalizar, demonstrar e expressar sentimentos de tristeza, medo e insatisfação, sendo $p<0,05$. Esses comportamentos foram verificados, principalmente, naquelas que relataram dor de forte intensidade, variando de $34,9 \%$ a $37,0 \%$, e dor leve, variando de $21,6 \%$ a $24,4 \%$. 
Tabela 1. Comportamento da criança, na sessão de brinquedo terapêutico, durante a troca de curativo pós-operatório conforme faixa etária, sexo e diagnóstico pré-operatório (CID-10)/procedimento

\begin{tabular}{|c|c|c|c|c|c|c|c|c|c|c|c|}
\hline \multirow{2}{*}{ Variáveis } & \multirow[b]{2}{*}{$\begin{array}{l}\text { Observa } \\
\text { n(\%) }\end{array}$} & \multirow[b]{2}{*}{$\begin{array}{c}\text { Manuseia } \\
\text { n(\%) }\end{array}$} & \multirow[b]{2}{*}{$\begin{array}{l}\text { Brinca } \\
\mathrm{n}(\%)\end{array}$} & \multirow[b]{2}{*}{$\begin{array}{c}\text { Interage } \\
\text { com adulto } \\
\text { n(\%) }\end{array}$} & \multicolumn{3}{|c|}{ Comportamento presente } & \multirow[b]{2}{*}{$\begin{array}{c}\text { Expressa } \\
\text { tristeza, } \\
\text { medo e } \\
\text { insatisfação } \\
\mathrm{n}(\%)\end{array}$} & \multirow[b]{2}{*}{$\begin{array}{c}\text { Compara } \\
\text { n(\%) }\end{array}$} & \multirow[b]{2}{*}{$\begin{array}{l}\text { Repete a } \\
\text { ação } \\
\text { n(\%) }\end{array}$} & \multirow[b]{2}{*}{$\begin{array}{c}\text { Movimentos } \\
\text { rituais } \\
\\
\\
n(\%)\end{array}$} \\
\hline & & & & & Verbaliza & Recusa & Demonstra & & & & \\
\hline \multicolumn{12}{|l|}{ Faixa etária } \\
\hline 4 a 6 anos & $47(94,0)^{* *}$ & $47(94,0)^{* *}$ & $26(52,0)^{* *}$ & $47(94,0)^{* *}$ & $47(94,0)^{* *}$ & $47(94,0)^{* *}$ & $49(98,0)^{* *}$ & $47(94,0)^{* *}$ & $15(30,0)^{* *}$ & $15(30,0) * *$ & $46(92,0)^{* *}$ \\
\hline$\geq 7$ anos & $36(59,7)$ & $26(41,9)$ & - & $27(43,5)$ & $27(43,5)$ & $3(4,8)$ & $37(59,7)$ & $30(48,4)$ & - & - & - \\
\hline \multicolumn{12}{|l|}{ Sexo } \\
\hline Feminino & $39(76,5)$ & $36(70,6)$ & $13(25,5)$ & $36(70,6)$ & $36(70,6)$ & $25(49,0)$ & $42(82,4)$ & $36(70,6)$ & $4(7,8)$ & $4(7,8)$ & $24(47,1)$ \\
\hline Masculino & $44(72,1)$ & $37(60,7)$ & $13(21,3)$ & $38(62,3)$ & $38(62,3)$ & $25(41,0)$ & $44(72,1)$ & $41(67,2)$ & $11(18,0)$ & $11(18,0)$ & $22(36,1)$ \\
\hline \multicolumn{12}{|l|}{$\begin{array}{l}\text { Diagnóstico pré- } \\
\text { operatório (CID-10)/ } \\
\text { procedimento }\end{array}$} \\
\hline $\begin{array}{l}\text { Apendicite (K36)/ } \\
\text { Apendicectomia }\end{array}$ & $14(38,9)^{* *}$ & $12(33,3)^{* *}$ & $7(19,4)^{* *}$ & $12(33,3)^{* *}$ & $12(33,3)^{* *}$ & $10(27,8)$ & $16(44,4)^{* *}$ & $14(38,9)^{* *}$ & $7(19,4)^{* *}$ & $7(19,4)^{* *}$ & $7(19,4)^{*}$ \\
\hline $\begin{array}{l}\text { Pneumotórax (J93)/ } \\
\text { Drenagem torácica }\end{array}$ & $17(89,5)$ & $16(84,2)$ & $6(31,6)$ & $17(89,5)$ & $17(89,5)$ & $11(57,9)$ & $17(89,5)$ & $17(89,5)$ & $3(15,8)$ & $3(15,8)$ & $11(57,9)$ \\
\hline $\begin{array}{l}\text { Hipospádia } \\
\text { peniana }(054.1) / \\
\text { Uretroplastia }\end{array}$ & $17(94,4)$ & $11(61,1)$ & - & $11(61,1)$ & $11(61,1)$ & $5(27,8)$ & $18(100,0)$ & $11(61,1)$ & - & - & $5(27,8)$ \\
\hline $\begin{array}{l}\text { Abscesso cutâneo } \\
\text { (L02.4)/ Drenagem } \\
\text { percutânea }\end{array}$ & $7(77,8)$ & $7(77,8)$ & $2(22,2)$ & $7(77,8)$ & $7(77,8)$ & $5(55,6)$ & $7(77,8)$ & $7(77,8)$ & - & - & $4(44,4)$ \\
\hline $\begin{array}{l}\text { Hérnia inguinal } \\
\text { bilateral (K40.0)/ } \\
\text { Herniorrafia }\end{array}$ & $9(100,0)$ & $9(100,0)$ & - & $9(100,0)$ & $9(100,0)$ & $6(66,7)$ & $9(100,0)$ & $9(100,0)$ & - & - & $6(66,7)$ \\
\hline $\begin{array}{l}\text { Obstrução } \\
\text { intestinal (K56.6)/ } \\
\text { Colostomia }\end{array}$ & $5(71,4)$ & $5(71,4)$ & $2(28,6)$ & $5(71,4)$ & $5(71,4)$ & $2(28,6)$ & $5(71,4)$ & $5(71,4)$ & - & - & $2(28,6)$ \\
\hline $\begin{array}{l}\text { Apendicite } \\
\text { com peritonite } \\
\text { generalizada } \\
\text { (K35.0)/ } \\
\text { Apendicectomia }\end{array}$ & $5(100,0)$ & $5(100,0)$ & $5(100,0)$ & $5(100,0)$ & $5(100,0)$ & $5(100,0)$ & $5(100,0)$ & $5(100,0)$ & $5(100,0)$ & $5(100,0)$ & $5(100,0)$ \\
\hline $\begin{array}{l}\text { Colecistite }(\text { K81)/ } \\
\text { Colecistectomia }\end{array}$ & $4(100,0)$ & $4(100,0)$ & $4(100,0)$ & $4(100,0)$ & $4(100,0)$ & $4(100,0)$ & $4(100,0)$ & $4(100,0)$ & - & - & $4(100,0)$ \\
\hline $\begin{array}{l}\text { Fimose (N47)/ } \\
\text { Postectomia }\end{array}$ & $3(100,0)$ & $3(100,0)$ & - & $3(100,0)$ & $3(100,0)$ & $1(33,3)$ & $3(100,0)$ & $3(100,0)$ & - & - & $1(33,3)$ \\
\hline Total & $83(100)$ & $73(100)$ & $26(100)$ & $74(100)$ & $74(100)$ & $50(100)$ & $86(100)$ & $77(100)$ & $15(100)$ & $46(100)$ & $46(100)$ \\
\hline
\end{tabular}

${ }^{*} p<0,05 ; * * p<0,001$

\section{Discussão}

Este estudo buscou analisar o comportamento e a dor de crianças no primeiro curativo pós-operatório, por meio da sessão de BT, com equipe técnica treinada para este fim. Aliviar a dor sempre foi considerado cuidado essencial à equipe de saúde, pois saná-la compreende direito humano básico. ${ }^{(9)}$ Contudo, por se tratar de fenômeno subjetivo, seu diagnóstico e mensuração dependem de instrumentos adaptados ao desenvolvimento comportamental e condição clínica de cada indivíduo. ${ }^{(10,11)}$
Pouca mais da metade das crianças desta pesquisa eram meninos, e tinham sete anos ou mais de idade. O procedimento cirúrgico mais observado foi apendicectomia, também referenciado em outra pesquisa, ${ }^{(3)}$ caracterizado como principal emergência de casos de abdome agudo, com maior prevalência em meninos. ${ }^{(12)}$ Neste estudo não houve diferença entre os sexos.

Complicações infecciosas precoces da apendicite aguda como formação de abscesso pélvico ou intra-abdominal, têm como consequências maiores incidências de episódios álgicos no pós-operatório e tempo de internação. ${ }^{(13)}$ Grande parte das crianças desta pesqui- 
Tabela 2. Relação do escore de dor segundo faixa etária, sexo, diagnóstico pré-operatório (CID-10)/procedimento e comportamento da criança

\begin{tabular}{|c|c|c|c|c|c|}
\hline Variáveis & $\mathrm{n}(\%)$ & $\mathrm{n}(\%)$ & $\mathrm{n}(\%)$ & $\mathrm{n}(\%)$ & $n(\%)$ \\
\hline Escore de dor & 0 & 1 & 2 & 3 & 4 \\
\hline \multicolumn{6}{|l|}{ Faixa etária } \\
\hline 4 a 6 anos & $3(6,0)$ & $13(26,0)$ & $8(16,0)$ & $17(34,0)$ & $9(18,0)$ \\
\hline$\geq 7$ anos & $11(17,7)$ & $13(21,0)$ & $7(11,3)$ & $13(21,0)$ & $18(29,0)$ \\
\hline \multicolumn{6}{|l|}{ Sexo } \\
\hline Feminino & $7(13,7)$ & $7(13,7)$ & $6(11,8)$ & $19(37,3)$ & $12(23,5)$ \\
\hline Masculino & $7(11,5)$ & $19(31,1)$ & $9(14,8)$ & $11(18,0)$ & $15(24,6)$ \\
\hline \multicolumn{6}{|l|}{ Diagnóstico pré-operatório (CID-10)/ procedimento } \\
\hline Apendicite (K36)/ Apendicectomia & $4(11,1)$ & $15(41,7)$ & $4(11,1)$ & $2(5,6)$ & $11(30,6)$ \\
\hline Pneumotórax (J93)/ Drenagem torácica & - & - & $3(15,8)$ & $6(31,6)$ & $10(52,6)$ \\
\hline Hipospádia peniana (Q54.1)/ Uretroplastia & $4(22,2)$ & $4(22,2)$ & - & $8(44,4)$ & $2(11,1)$ \\
\hline Abscesso cutâneo (L02.4)/ Drenagem percutânea & $2(22,2)$ & - & $3(33,3)$ & $4(44,4)$ & - \\
\hline Hérnia inguinal bilateral (K40.0)/ Herniorrafia & $2(22,2)$ & $2(22,2)$ & - & $5(55,6)$ & - \\
\hline Obstrução intestinal (K56.6)/ Colostomia & $1(14,3)$ & $2(28,6)$ & $2(28,6)$ & $1(14,3)$ & $1(14,3)$ \\
\hline Apendicite com peritonite generalizada (K35.0)/ Apendicectomia & - & - & - & $2(40,0)$ & $3(60,0)$ \\
\hline Colecistite $($ K81)/ Colecistectomia & - & $1(25,0)$ & $2(50,0)$ & $1(25,0)$ & - \\
\hline Fimose (N47)/ Postectomia & - & $2(66,7)$ & $1(33,3)$ & - & - \\
\hline \multicolumn{6}{|l|}{ Comportamento } \\
\hline Observa** & $7(8,4)$ & $19(22,9)$ & $11(13,3)$ & $30(36,1)$ & $16(19,3)$ \\
\hline Manuseia** & $6(8,2)$ & $16(21,9)$ & $11(15,1)$ & $27(37,0)$ & $13(17,8)$ \\
\hline Brinca & - & $9(34,6)$ & $5(19,2)$ & $6(23,1)$ & $6(23,1)$ \\
\hline Interage com 0 adulto** & $6(8,1)$ & $16(21,6)$ & $11(14,9)$ & $27(36,5)$ & $14(18,9)$ \\
\hline Verbaliza** & $6(8,1)$ & $16(21,6)$ & $11(14,9)$ & $27(36,5)$ & $14(18,9)$ \\
\hline Recusa & $3(6,0)$ & $11(22,0)$ & $8(16,0)$ & $19(38,0)$ & $9(18,0)$ \\
\hline Demonstra** & $8(9,3)$ & $21(24,4)$ & $11(12,8)$ & $30(34,9)$ & $16(18,6)$ \\
\hline Expressa tristeza, medo e insatisfação** & $7(9,1)$ & $17(22,1)$ & $11(14,3)$ & $27(35,1)$ & $15(19,5)$ \\
\hline Compara & - & $7(46,7)$ & - & $4(26,7)$ & $4(26,7)$ \\
\hline Repete a ação & - & $7(46,7)$ & - & $4(26,7)$ & $4(26,7)$ \\
\hline Movimentos rituais & $3(6,5)$ & $11(23,9)$ & $8(17,4)$ & $16(34,8)$ & $8(17,4)$ \\
\hline Total & $14(100)$ & $26(100)$ & $15(100)$ & $30(100)$ & $27(100)$ \\
\hline
\end{tabular}

*Excluídos registros com informações ignoradas; **p<0,05

sa submetidas à apendicectomia, principalmente com peritonite generalizada, apresentaram dor de intensidade moderada a insuportável, além de menos interação com o boneco durante a sessão do BT.

A drenagem torácica, procedimento de grande prevalência nesta amostra, é opção terapêutica para pneumonias complicadas e frequente motivo de internação em crianças, especialmente nas que possuem menos de três anos de idade. Nessas situações, um tratamento adequado voltado à fase evolutiva do derrame pleural previne internação prolongada e complicações tardias, como encarceramento pulmonar, proporcionando melhor qualidade da assistência e menor sofrimento infantil. Ao se reduzir o desgaste físico, principalmente evitando-se dor pós-operatória, introdução de drenos torácicos, curativos nas inserções dos dispositivos médicos e manobras torácicas de profissionais especializados, como fisioterapeutas respira- tórios, por exemplo, também se reduz o sofrimento psicológico da criança e sua família, minimizando os impactos negativos da hospitalização sobre seu desenvolvimento. ${ }^{(14)}$ As crianças submetidas à esse procedimento cirúrgico também relataram dor de intensidade moderada a insuportável, com elevada prevalência de expressão de tristeza, medo e insatisfação durante a interação com o boneco na sessão de BT.

A uretroplastia, terceiro procedimento mais frequente observado nesta pesquisa, foi decorrente de hipospádia peniana. Trata-se de defeito anatômico congênito mais comum da uretra masculina, resultado de fatores genéticos e/ou endócrinos. ${ }^{(15,16)}$ Achado semelhante foi verificado em outra pesquisa cujo objetivo foi caracterizar o perfil das cirurgias em unidade pediátrica de hospital público, com 10,8\% das internações por essa causa. ${ }^{(3)}$ As crianças submetidas à uretroplastia, desta pesquisa, apresentaram segunda menor 
prevalência dos comportamentos analisados durante a sessão de BT, destacando-se apenas o ato de observar. A limitação física do pós-operatório para otimização da recuperação cirúrgica, associada à dor que se agrava durante o primeiro curativo pela sensibilidade local, pode alterar o humor da criança e dificultar o manejo terapêutico por parte da equipe de saúde, gerando ansiedade e medo à aproximação de pessoas que não são de seu convívio familiar.

As crianças menores de sete anos de idade foram as que mais apresentaram diversidade de comportamentos durante a sessão de BT, ressaltando-se o ato de demonstrar, no boneco, a realização do primeiro curativo pós-operatório ao qual havia sido recentemente submetida. Destacaram-se, também, comportamentos de observar o cenário do BT, manusear os brinquedos e materiais hospitalares, interagir com adulto, verbalizar as percepções vivenciadas a partir do uso do boneco, recusar a realização do curativo em forma de brincadeira, demonstrar o procedimento, expressar tristeza, medo e insatisfação, e realizar movimentos rituais de defesa durante a sessão de BT. Comportamentos semelhantes foram verificados em estudo que avaliou as memórias pós-cirúrgicas em crianças submetidas às cirurgias eletivas. ${ }^{(5)}$

A população infantil apresenta estratégias de enfrentamento mais específicas que o adulto, reflexo dos desenvolvimentos cognitivo e social, e de regulação da emoção inerente à idade. Crianças pequenas, presas a um pensamento concreto, se beneficiam de enfrentamentos comportamentais e buscam por apoio emocional. Já crianças mais velhas e jovens são capazes de controlar emoções e usar recursos imaginários como, por exemplo, praticar o relaxamento ou transformar o significado subjetivo da situação estressante. (5,7,17) $^{(17}$

Pesquisa para conhecer estratégias de enfrentamento de crianças de sete a 11 anos, hospitalizadas em situação pré-cirúrgica, e de seus acompanhantes, identificou presença de estresse em 50,0\% delas, enquanto nas mães esse percentual totalizou $77,6 \%$. Maiores dificuldades comportamentais e menores estratégias de reestruturação cognitiva, ou seja, capacidade de transformar pensamentos desadaptativos e disfuncionais em algo positivo e significativo sob nova perspectiva, foram observadas nas crianças de sete a oito anos comparada às mais velhas. ${ }^{(17)}$ Além disso, a experiência cirúrgica prévia favoreceu esse processo de reestruturação cognitiva em relação àquelas que passavam pelo procedimento pela primeira vez. Crianças cujos acompanhantes pontuaram elevados índices de estresse também demonstraram maiores estratégias de regulação da emoção e da ruminação (pensamentos negativos e recorrentes) que crianças de cuidadores sem estresse. ${ }^{(17)}$

Outros fatores devem ser considerados pela equipe de saúde na avaliação do comportamento da criança que vão além da idade, como temperamento, possibilidade de controle do agente estressor, experiência álgica prévia, cirurgias e procedimentos invasivos, modelos de enfrentamento familiar e reação dos pais às situações adversas. ${ }^{(7,18)}$ Torna-se, portanto, essencial a coparticipação parental na assistência pré, trans e pós-operatória para promoção da saúde e recuperação infantis.

Memórias pós-operatórias são fidedignas ao contexto cirúrgico ao qual são submetidas. ${ }^{(5)}$ Elas recordam daquilo que vivenciaram e presenciaram desde sua chegada no hospital até o momento da alta, retratando sentimentos sobre a internação e cirurgia de forma detalhada. ${ }^{(5-7)}$ Uma estratégia que pode ser utilizada pelas unidades pediátricas para qualificação da assistência e promoção do bem-estar infantil é a aplicação do BT. Revisão sistemática realizada entre 1983 e 2015, mostrou que essa intervenção promove redução nos níveis de ansiedade e estresse, e favorece comportamento de colaboração junto à equipe de saúde, porém novos estudos com rigor metodológico devem ser estimulados para comprovação de sua eficácia. ${ }^{(19)}$

O comportamento da criança pode variar de acordo com a experiência dolorosa que é submetida, podendo tornar-se introspectivas e solitárias no imaginário infantil. Neste estudo, a dor sentida durante a troca do primeiro curativo pós-operatório classificada como de intensidade insuportável inibiu várias expressões comportamentais, no boneco, durante a sessão de BT. A análise comportamental nessas situações permitem intervenções farmacológicas e não farmacológicas prévias que garantam maior segurança e suporte emocional, protegendo-as das sensações temerosas e aflituosas.(2)

A dor pós-cirúrgica reúne diversas experiências sensoriais, emocionais e mentais desagradáveis, associadas às respostas autonômicas, endócrinas, metabólicas, fisiológicas e comportamentais. Segundo a In- 
ternational Association for the Study of Pain (IASP), mais de $80 \%$ dos pacientes submetidos à cirurgia relataram dor no pós-operatório. ${ }^{(20)}$ Nesta pesquisa, cerca de $83,0 \%$ das crianças referiram dor durante o curativo, sendo $61,5 \%$ de intensidade moderada a insuportável. As crianças normalmente possuem mesmo escore de dor pós-operatória que os adultos, embora a cicatrização seja mais rápida. Contudo, é uma população peculiar pela complexidade da manifestação do processo álgico, em que mecanismos de autoproteção costumam ser ineficazes e a não verbalização desafiam os profissionais da saúde no reconhecimento, avaliação e tratamento adequados da dor. ${ }^{(21)}$

O não alívio da dor gera consequências de curto e longo prazos. No primeiro pode haver aumento dos riscos de morbidade e mortalidade pós-operatória, e prolongamento do tempo de internação com consequente elevação dos custos hospitalares. No segundo não é raro observar complicações como dor crônica, que costuma incidir em 10,0 a 50,0\% dos pacientes que se submeteram a procedimentos cirúrgicos comuns, sendo de intensidade severa em 2,0 a 10,0\% dos casos, e costumam cursar com quadros de atraso do desenvolvimento, baixo rendimento escolar e depressão. ${ }^{(20)}$ O controle ineficaz da dor aguda pós-operatória pode estar associado à futura existência de dor crônica, ou seja, com duração igual ou superior a três meses. ${ }^{(20)}$

Cirurgias e hospitalizações privam crianças de seu cotidiano e produzem experiências dolorosas e desagradáveis que potencializam surgimento de variados sentimentos, como culpa, ansiedade e medo do desconhecido e da morte. ${ }^{(22)} \mathrm{O}$ BT é muito importante nesse processo, e deve ser realizado por profissional capacitado para esse fim. ${ }^{(22)} \mathrm{O}$ enfermeiro é o profissional que atua afetivamente nessas situações, protegendo a criança de sentimentos degradantes e propondo terapias não farmacológicas coadjuvantes no combate a dor como risoterapia, musicoterapia, exercícios de relaxamento e respiração, e apoio psicológico com profissionais habilitados. ${ }^{(23)}$

Esta pesquisa vem de encontro a necessidade de estudos que aprofundem a análise do comportamento e da dor de crianças cirúrgicas durante a realização do primeiro curativo pós-operatório. Contudo trata-se de estudo transversal, não intervencionista, em uma amostra de conveniência, não devendo os resultados serem extrapolados à população geral. O número re- duzido de crianças analisadas, assim como o não uso de ajustes estatísticos por variáveis confundidoras, como classe de analgésicos prescritos, periodicidade de administração e uso durante a realização do procedimento, podem ter repercutido em algumas análises. Novos estudos longitudinais, intervencionistas e com maior número de participantes devem ser estimulados para demonstrar a eficácia do BT no preparo prévio da criança a evento traumático, como por exemplo um procedimento cirúrgico, e na modulação do comportamento infantil e da dor para redução de medos, ansiedades e estresse dos núcleos familiares. Espera-se que os achados desta pesquisa possam auxiliar no planejamento de futuras abordagens, assim como permitir reflexão sobre o cuidado da criança pós-cirúrgica em situações adversas.

\section{Conclusão}

A prevalência de dor no primeiro curativo pós-operatório foi elevada, com destaque àquela mensurada de intensidade moderada a insuportável. Crianças com menos de sete anos participaram mais na sessão do BT, demonstrando comportamentos variados positivos e negativos. As que estavam em pós-operatório de apendicectomia interagiram pouco. Esse também foi um dos curativos mais doloridos reportados nesta população. Inúmeras são as estratégias farmacológicas e não farmacológicas disponíveis em ambiente hospitalar para controle da dor, as quais devem ser avaliadas e utilizadas conforme a individualidade da criança e família com objetivo de melhorar a assistência prestada e garantir mais segurança e conforto durante a hospitalização. O uso do BT deve ser cada vez mais estimulado ao se considerar a diversidade de suporte psicológico que é capaz de proporcionar, iniciando pelo preparo da criança até extravasamento de sentimentos desadaptativos e disfuncionais, com diminuição de traumas e impactos destrutivos de experiências adversas ao desenvolvimento infantil.

\section{Contribuições}

Miranda LL, Tacla MT, Gabani FL e Ferrari RA declaram que contribuíram com a concepção do estudo, 
análise e interpretação dos dados, redação do artigo, revisão crítica relevante do conteúdo intelectual e aprovação da versão final a ser publicada.

\section{Referências}

1. Farias DD, Gabatz RI, Terra AP, Couto GR, Milbrath VM, Schwartz E. A hospitalização na perspectiva da criança: uma revisão integrativa. Rev Enferm UFPE. 2017;11(2):703-11.

2. Fernandes SC, Arriaga P, Esteves F. Atitudes infantis face aos cuidados de saúde e percepção de dor: papel mediador dos medos médicos. Ciênc Saúde Coletiva. 2014;19(7):2073-82.

3. Alves BA, Santos TF, Ferrari RA, Tacla MT, Sant'Anna FL, Lopes EB. Criança hospitalizada: caracterização dos procedimentos cirúrgicos em um hospital escola público. Semina Cienc Biol Saude. 2015;36(1):317-24.

4. Santos JP, Maranhão DG. Cuidado de enfermagem e manejo da dor em crianças hospitalizadas: pesquisa bibliográfica. Rev Soc Bras Enferm Ped. 2016;16(1):44-50.

5. Broering CV, Crepaldi MA. 0 estudo das memórias pós-cirúrgicas: importância e limitações. Vittalle. 2013;25(2):53-61.

6. Sng QW, Taylor B, Liam JL, Klainin-Yobas P,Wang W. He HG. Postoperative pain management experiences among school-aged children: a qualitative study. J Clin Nurs. 2013;22:958-68.

7. Pfeifer PM, Quintana AM. 0 ato cirúrgico e as fantasias infantis: uma revisão da literatura. Mudanças - Psicologia da Saúde. 2015;23(2):9-16.

8. Lerwick JL. Psychosocial implications of pediatric surgical hospitalization. Semin Pediatr Surg. 2013; 22(3):129-33.

9. Ribeiro JP, Gomes GC, Thofehrn MB. Ambiência como estratégia de humanização da assistência na unidade de pediatria: revisão sistemática. Rev ESc Enferm USP. 2014;48(3):530-9

10. Azevedo DM, Nascimento VM, Azevedo IC, Cavalcanti RD, Sales LK. Assistência de enfermagem à criança com dor: avaliação e intervenções da equipe de enfermagem. Rev Bras Pesq Saúde. 2014;16(4):23-31.
11. Ávila MJ, García-Acero M. Apendicitis aguda: revisión de la presentación histopatológica en Boyacá, Colombia. Rev Colomb Cir. 2015;30:125-30.

12. Iamarino AP, Juliano Y, Rosa OM, Novo NF, Favaro ML, Ribeiro Júnior MA. Fatores de risco associados às complicações de apendicite aguda. Rev Col Bras Cir. 2017;44(6):560-66.

13. Brasil. Conselho Federal de Enfermagem. Resolução COFEN $n^{0} 295$, de 24 de outubro de 2004: dispõe sobre a utilização da técnica do brinquedo/brinquedo terapêutico pelo enfermeiro na assistência à criança. Rio de Janeiro (RJ): Conselho Federal de Enfermagem; 2004.

14. Amorim LE, Camargos PA, Almeida MB, Gomide LD, Pereira GT, Ibiapina CC. Abordagem do derrame pleural parapneumônico em crianças sob a forma de mapa conceitual. Rev Med Minas Gerais. 2016;26(Supl 6):38-43.

15. George M, Schneuer FJ, Jamieson SE, Holland AJ. Genetic and environmental factors in the aetiology of hypospadias. Pediatr Surg Int. 2015;31(6):519-27.

16. Nogueira PC, Paz IP. Sinais e sintomas das anormalidades do desenvolvimento do trato geniturinário. J Pediatr. 2016:92(1):57-63.

17. Carnier LE, Padovani FH, Perosa GB, Rodrigues OM. Estratégias de enfrentamento em crianças em situação pré-cirúrgica: relação com idade, sexo, experiência com cirurgia e estresse. Estud Psicol. 2015;32(2):319-30.

18. Papalia DE, Feldman RD, Martorell G. Desenvolvimento humano. 12ª ed. São Paulo: AMGH Editora; 2013.

19. Silva RD, Austregésilo SC, Ithamar L, Lima LS. Therapeutic play to prepare children for invasive procedures: a systematic review. J Pediatr. 2017;93(1):6-16.

20. International Association for the Study of Pain. Postoperative pain in children and babies. [2020 July 17]. Available from: http://www.aped-dor.org/GY_GY_2017/7.Dorpediatrica. Finley-Pt.pdf

21. Finley GA, Franck LS, Grunau RE, Baeyer CL. Why children's pain matters. Pain: Clinical Updates. 2005;13(4):1-6.

22. Cardoso NR, Prado PF, Souza AA, Figueiredo ML. Vivenciando o processo cirúrgico: percepção e sentimentos da criança. Rev Baiana Enferm. 2017;31(3):e17648.

23. Gomez-Torres D, Maldonado-González V; Reyes-Robles B, Carrera AL. Atuação humanizada da enfermeira diante a dor do paciente infantil queimado. Cogitare Enferm. 2014;19(2):246-53.

24. Castral TC, Daré MF, Scochi CG. Prioridades de pesquisa em enfermagem neonatal e pediátrica. Rev Eletr Enf. 2014;16(1):12-4. 